\title{
Interactive comment on "Integration of Airborne and Ground Observations of Nitryl Chloride in the Seoul Metropolitan Area and the Implications on Regional Oxidation Capacity During KORUS-AQ 2016" by Daun Jeong et al.
}

Daun Jeong et al.

saewungk@uci.edu

Received and published: 17 April 2019

Please see an attached file containing our line-by-line responses to the comments

Please also note the supplement to this comment:

https://www.atmos-chem-phys-discuss.net/acp-2018-1216/acp-2018-1216-AC1- 
2018.

ACPD

Interactive

comment

Printer-friendly version

Discussion paper 